\title{
Structure and Crystallography of Second Order Twins in Copper
}

\author{
by M. Sharp and C. G. Dunn
}

$I_{n}^{N}$ $\mathrm{N}$ twinned crystals of the face-centered cubic metals the lattice of one twin is a mirror image of the other in a common twin boundary. When several twins appear within one large grain in a sheet specimen, the twin boundaries form a set of lines at the surface of the specimen which coincide with $\{111\}$ planes of the large grain. Furthermore, for twins of the same orientation, these lines are parallel.

Generally, the presence of identically oriented regions with straight parallel boundaries coinciding with a $\{111\}$ plane of the surrounding crystal is strong evidence for identifying the island regions as twins of the parent crystal. However, Fig. 1, which shows the macrostructure of a large grain of copper with island regions that satisfy these conditions, is not an illustration of $\{111\}$ twins. Since

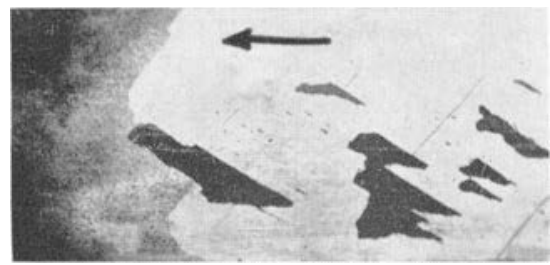

Fig. 1-Macrograph of a large grain of copper with several relatively large second-order twins. Natural size.

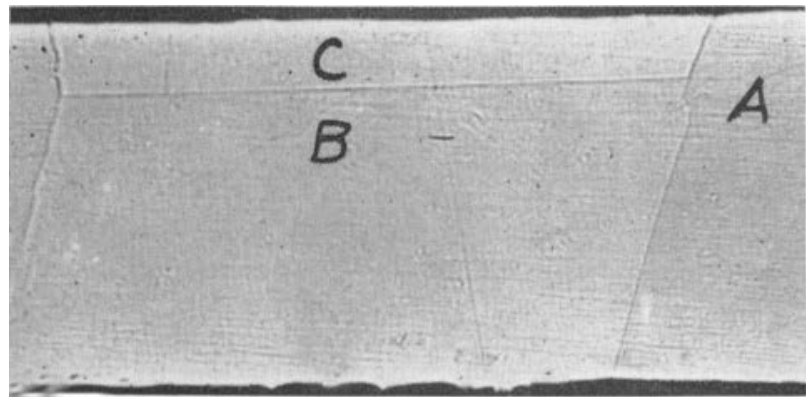

Fig. 2-Micrograph of section taken at point of initial growth of first-order twin $B$ and second-order twin $C$. The section is cut perpendicular to both the surface of the sample and the straight boundary between grains $A$ and $C$. X100. Area reduced approximately 50 pet for reproduction.

the reverse side of the specimen has much the same appearance, it was thought at first that these regions, which appear dark in the macrograph, actually were twins. According to X-ray data, however, these regions are second-order twins of the large crystal.

With regard to their formation, these secondorder twins formed by secondary recrystallization in a cube texture matrix. Growth occurred in the direction of the arrow (see Fig. 1) as the specimen moved slowly into a gradient temperature furnace as described previously. ${ }^{1}$ Nucleation of the

M. SHARP, Junior Member AIME, is with the Chrysler Jet Engine Plant, Detroit, Mich., and C. G. DUNN, Member AIME, is associated with General Electric Co., Pittsfield, Mass.

TN 125E. Manuscript, June 28, 1952.

This work was supported by the U. S. Atomic Energy Commission under Contract No. W-31-109-Eng-52. second-order twins occurred, therefore, on the ends facing opposite the arrow. If the origin of the second-order twins were due to repeated twinning, some first-order twin structure should be visible on these ends. This proved to be the case, as very small twins were readily found with the aid of a microscope, and probably could have been seen, in some instances, under ideal lighting conditions without aid of a microscope.

Fig. 2 shows a cross-section view taken perpendicular to both the surface and the $\{111\}$ trace of the parent crystal (visible as a straight boundary in Fig. 1) at the beginning point of growth of a second-order twin and where one first-order twin was relatively thick. In the micrograph, $A$ is the large parent grain; $B$ is the first-order twin of $A$; and $C$, which is a first-order twin of $B$, is a secondorder twin of $A$. Between $A$ and $B$ and between $B$ and $C$ the major straight portions are traces of common $\{111\}$ twin boundaries. The straight portion of boundary between $A$ and $C$, however, is not a common crystallographic plane to the two lattices; it is a $\{111\}$ plane of $A$ and a $\{115\}$ plane of $C$.

Without considering the mechanism of twinning itself, the origin of the second-order twins may be accounted for in terms of repeated twinning and special growth characteristics. After each nucleation, a selective growth process can be thought of as favoring growth of the first-order twin in local spots only and favoring growth of the second-order twin to an extent comparable with that of the parent grain over relatively large areas in a way similar to that described for twinning in aluminum. ${ }^{2}$

It has already been pointed out that the boundary between the large grain $(A)$ and the second-order twin (C), which is responsible for the straight boundary portions in Fig. 1, involves a $\{111\}$ plane of $A$ and a $\{115\}$ plane of $C$. The same combination of planes is not only possible in first-order twins, but actually appears quite frequently. ${ }^{8}$ Their prevalence in first-order twins and their presence here in second-order twins, together with the necessary occurrence of a large number of common lattice sites at the boundary, is an indication that this combination produces an "energy cusp"" boundary. (Energy cusp boundaries have been described by Shockley and Read. $\left.{ }^{4}\right)$ The configuration of atoms near a $\{111\}$, $\{115\}$ boundary in first-order twins is of course different from the configuration near the same type of boundary in second-order twins.

\section{References}

${ }^{1}$ M. Sharp and C. G. Dunn: Secondary Recrystallization Texture in Copper. Journal of Metals (January 1952) Trans. AIME, p. 42.

${ }^{2}$ W. G. Burgers and W. May: Stimulated Crystals and Twinning in Recrystallized Aluminum. Recueil des travaux chimiques des Pays-Bas (1945) 64, p. 5.

${ }^{3} \mathrm{D}$. Whitwham, M. Mouflard, and P. Lacombe: Discussion of W. C. Ellis and R. G. Treuting, "Atomic Relationships in the Cubic Twinned State." Trans. AIME (1951) 191, p. 1070; Journal of Metals (October 1951).

${ }^{4}$ W. Shockley and W. T. Read: Dislocation Models of Crystal Grain Boundaries. Physical Review (1950) 78 , p. 275. 